Egyptian Journal of Aquatic Biology \& Fisheries

Zoology Department, Faculty of Science,

Ain Shams University, Cairo, Egypt.

ISSN $1110-6131$

Vol. 25 (4): 211 - 220 (2021)

www.ejabf.journals.ekb.eg

\title{
Morphological and molecular characterizations of two Acanthamoeba spp. from tap water in Nasr City, Cairo Governorate, Egypt
}

\author{
Yasmen A. Abdelaziz ${ }^{1}$, Ahmed Abd El-Aziz ${ }^{2}$, Wafaa F.A. Ahmed ${ }^{1}$, \\ Mohamed F. Abou El-Nour ${ }^{2}$ \\ ${ }^{1}$ Biological and Environmental Sciences Dept., Faculty of Home Economics, Al-Azhar University, Egypt \\ ${ }^{2}$ Zoology Department, Faculty of Science, Al-Azhar University, Nasr City, 11884, Cairo, Egypt
}

Corresponding Author: safa.hamza92@yahoo.com

\begin{abstract}
ARTICLE INFO
Article History:

Received: July 4, 2021

Accepted: July 28, 2021

Online: July 30, 2021

Keywords:

Acanthamoeba;

Morphological;

Molecular;

Characterization

\section{ABSTRACT}

Providing suitable drinking water for human use is a major goal in all countries, and contamination of drinking water with microorganisms represents a great danger to human health. Free-living amoebae (FLAs, Pathogenic) are found in all natural and manufactured environments, including aquatic environments. Several FLAs such as Acanthamoeba spp. infect humans, causing dangerous diseases. The purpose of this investigation was to search about FLAs in tap water in Nasr City, Cairo. FLAs were detected in $59(53.63 \%)$ out of 110 tap water samples which were morphologically identified as two Acanthamoeba spp. The molecular identification to the species level was carried out, using a simple PCR technique and DNA sequencing. Acanthamoeba spp. were recorded in the gene bank under accession numbers Acanthamoeba MT944118 and Acanthamoeba MT944119.
\end{abstract}

\section{INTRODUCTION}

Safe and readily available water is important for human public health, whether it is used for drinking, domestic use, food production or recreational purposes. Improved water supply and better management of water resources can boost countries' economic growth and can contribute greatly to poverty reduction. Contamination of drinking water with microorganisms also, represents a great danger to human health in many parts of the world (Trabelsi et al., 2012). Free-living amoebae are numerous and systematically heterogeneous protozoan groups with worldwide distribution including water, soil, dust, and air. They have been isolated from natural water environments such as rivers, lakes, springs, and man-made water systems as tap water systems (even in hospitals), cooling towers, water tanks, wastewater treatment plants, pharmaceutical factory drains, swimming pools, sewages, contact lens cases, air conditioning equipment, dialysis units, dental treatment units and others (Bandirzadeh et al., 2011).

Acanthamoeba spp. were the most detected pathogenic FLAs in the environment. They have a two stages - life cycle which includes a vegetative form (trophozoite) as a motile trophic stage, when the amoeba is in a humid, favorable and nutrition rich environment. When not adherent to a surface, they can also form so called swimming forms showing their typical acanthopodia (Anwar et al., 2019). Acanthamoeba species become dormant and enter the cyst 
stage as soon as the conditions become not favorable. These cysts are resistant to a certain degree to environmental or manmade stress (e.g., disinfection measures or medical treatment). They can tolerate a wide range of environmental conditions. As some Acanthamoeba strains are thermotolerant, they are capable of infecting humans (Shyrobokov et al., 2020).

The first suggestion that Acanthamoeba spp. could cause disease in human came in 1958 during polio vaccine safety trials (Culbertson et al., 1958 \&1959). Whereas Acanthamoeba sp. was first recognized in a human as an ocular pathogen in 1973 and was caused an epidemic in the 1980s due to the contaminated contact lenses (O'Day and Head, 2000). Amebic keratitis (AK), a painful sight-threatening disease of the eyes was caused by Acanthamoeba species (Martinez and Visvesvara, 1997). Human cases of granulomatous amebic encephalitis (GAE), a fatal disease of central nervous system (CNS) caused also by Acanthamoeba species, were reported from Australia, Europe, Africa, South America, and United States (Rodriguez-Zaragoza, 1994). Acanthamoeba species also have been associated with cutaneous lesions and sinusitis in AIDS patients and other immunocompromised individuals (Neelam \& Niederkorn, 2017).

Besides their own pathogenic ability, some FLAs may serve as reservoirs of many intracellular pathogenic bacteria such as Legionella pneumophila, Mycobacterium spp. and Pseudomonas spp., as well as viruses (Greub \& Raoult, 2004). Consequently, the interest in both FLAs and the association of microorganisms with FLAs has significantly increased in the last years in relation to the risk to human public health (Scheid, 2007). Furthermore, Acanthamoeba spp. may have veterinary significance as demonstrated by their presence in diseased or dead cows, dogs, pigs, rabbits, pigeons, sheep, reptiles, fish, turkeys, horses, and other animals (Kinde et al., 2007).

Aim of the study was to investigate the presence of pathogenic free-living amoebae in tap water samples collected from some regions in Nasr City, Cairo, Egypt. Morphological description and identification of the isolated FLAs have been carried out, followed by a molecular study, using a simple PCR technique and sequencing, to confirm and to identify the isolated FLAs to the species level.

\section{MATERIALS AND METHODS}

\section{Water samples collection}

A total of 110 tap water samples (each water sample one-liter volume) were collected from some regions in Nasr City, Cairo, Egypt from December 2017 to September 2018. Samples were collected in clean, dry autoclavable polypropylene containers, and transported in an ice box to Invertebrate and Parasitology Lab., Zoology Department, Faculty of Science (Boys), Al-Azhar University, Nasr City, Cairo, Egypt. Samples were processed at the same day of collection.

\section{Samples' concentration, and cultivation and isolation of FLAs}

At the lab., tap water samples were centrifuged at $2000 \mathrm{rpm}$ for $15 \mathrm{~min}$. One or two drops of sediment samples were spread onto the Non-Nutrient Agar (NNA) plates coated with a layer of Escherichia coli (E. coli). The plates were then, incubated at room temperature $\left(25-28^{\circ} \mathrm{C}\right)$ for one week (Abdul Majid $\boldsymbol{e t}$ al., 2017). Existence of pathogenic free-living amoebae could be tracked by the clear tracks on $E$. coli lawn NNA produced by the feeding trophozoites. 
Escherichia coli strain (NCTC 12241/ATCC ® 25922) was obtained from American Type Culture Collection, USA. A typical E. coli colony was inoculated into a nutrient broth tube at $37^{\circ} \mathrm{C}$. After $24 \mathrm{~h}$. of incubation, E. coli was ready to be used as a food source for the cultured amoebae (APHA, 2005). Every 4 days, E. coli culture was maintained by sub-culturing in fresh nutrient broth tubes that were incubated at the same conditions, as mentioned before.

\section{Morphological identification of the isolated FLAs}

The isolated and purified FLAs from tap water were morphologically identified on bases of the characteristics of both trophozoites and cysts according to Pussard and Pons (1977) and Page (1988).

\section{Molecular study}

A simple PCR technique, consisting of 3 steps: DNA extraction, amplification, and agarose gel electrophoresis, was done. DNA was extracted from the obtained amoebae using a QIAGEN@, DNA Mini-Prep Kit (Madison, USA). Genus-specific primer and its fragments of target genes were used for Acanthamoeba spp. (as morphologically identified). Extracted DNA was amplified using foreword primer 5'-CCCAGATCGTTTACCGTGAA-3' and reverse primer 5'TAAATATTAATGCCCCCAACTATCC-3', according to the method of Schroeder et al. (2001). Electrophoresis was carried out using 1\% agarose gel to separate DNA fragments as described by Helling et al. (1974). PCR conditions were as follows: an initial denaturing step of $3 \mathrm{~min}$ at $95^{\circ} \mathrm{C}$, followed by 40 cycles of one min at $95^{\circ} \mathrm{C}$, one min at $55^{\circ} \mathrm{C}$, and two min at $72^{\circ} \mathrm{C}$. These were followed by a final extension of ten min at $72^{\circ} \mathrm{C}$. Amplicons of approximately 450 bp for Acanthamoeba spp. were visualized on a 1\% agarose gel and photographed using a gel documentation system. PCR positive samples for Acanthamoeba were subjected to DNA sequencing using the ABI PRISM® automated DNA Sequence. Nucleotide sequences were analyzed and assembled using the Laser gene 6 Package ${ }^{\circledR}$ (DNASTAR) and BLAST analysis tools (http://www.ncbi.nlm.gov/BLAST). The sequences were prepared and aligned using CLUSTAL W implemented in the Bio-Edit program (version 7.0.4.1) (Kumar et al., 2016).

\section{RESULTS}

\section{Isolation and identification of FLAs}

110 tap water samples collected from some regions in Nasr City, Cairo, Egypt were carefully examined microscopically for the presence of free-living amoebae. FLAs were detected in $59(53.63 \%)$ out of 110 samples. Morphological identification based on the characteristics of both trophozoites and cysts of amoebae, showed that only two different Acanthamoeba spp.: Acanthamoeba sp. A and Acanthamoeba sp. B were observed during the study.

\section{Characteristics of Acanthamoeba sp. A Acanthamoeba sp. A trophozoite}

Acanthamoeba sp. A trophozoite (Fig. 1) was 20-24 $\mu \mathrm{m}$ in size. The outline was often irregular, but it was generally longer than broad. A single vesiculate nucleus measured $4-8 \mu \mathrm{m}$ in diameter had a characteristically large centrally located dense nucleolus, surrounded by a clear 
halo and a thin nuclear membrane. More than contractile vacuoles were observed. Trophozoite had slender finger-like projections (acanthopodia) arising from the cytoplasm.

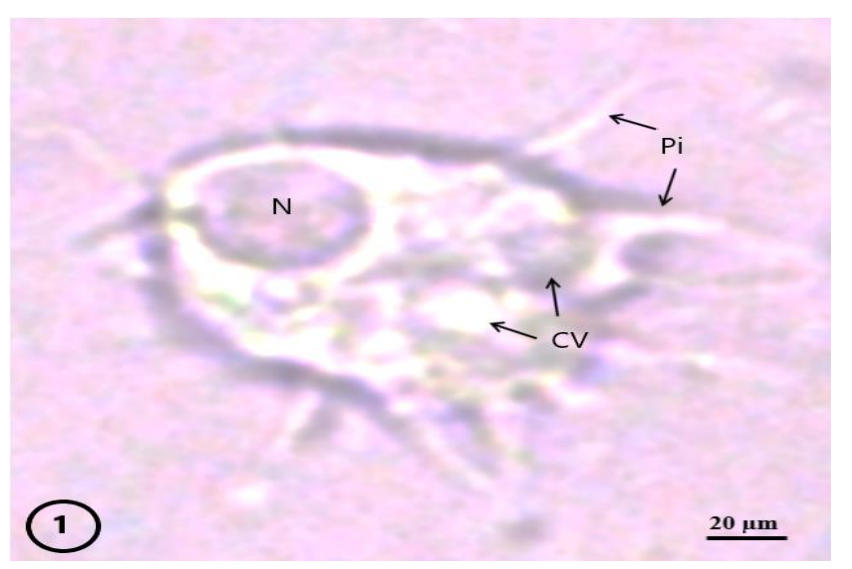

Fig. (1): Photomicrograph of Acanthamoeba sp. A trophozoite, stained with Lugol's iodine. It had an irregular outline; Vesicular nucleus $(N)$ had a large centrally located dense nucleolus surrounded by a clear halo and thin nuclear membrane. Note that more than contractile vacuole (CV) and slender finger-like cytoplasmic projections (acanthopodia) (Pi) were seen.

\section{Acanthamoeba sp. A cyst}

Acanthamoeba sp. A cyst (Fig. 2) tended to have a greatly irregular shape (polygonal). Cyst was characterized by the presence of a double wall: a smooth or wrinkled outer wall, ectocyst, which was moderately thin, with a little dilation, and an inner wall, endocyst, which had the same thickness of ectocyst. Pores were $4-5$ in number. Acanthamoeba sp. A cyst was $14 \pm$ $1.5 \mu \mathrm{m}$ in size.

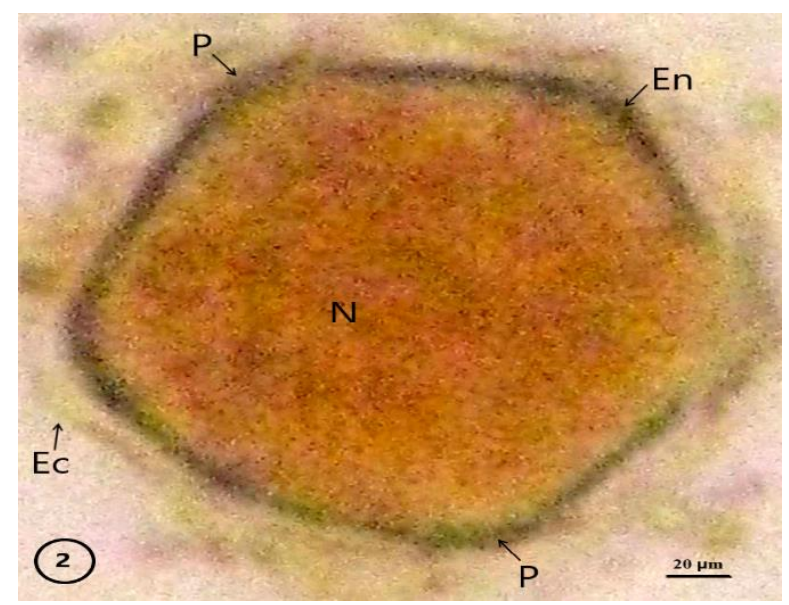

Fig. (2): Photomicrograph of Acanthamoeba sp. A cyst, stained with Lugol's iodine. It had a single nucleus (N), a smooth or wrinkled outer wall ectocyst (Ec) and a polygonal wall endocyst (En) with pores (P). 
Characteristics of Acanthamoeba sp. B

Acanthamoeba sp. B trophozoite

Trophozoite of Acanthamoeba sp. B was $25-30 \mu \mathrm{m}$ in size and had often an irregular outline (Fig. 3). A single vesiculate nucleus measured 5-10 $\mu \mathrm{m}$ in diameter, had a characteristically large centrally located dense nucleolus, surrounded by a clear halo and a thin nuclear membrane. More than contractile vacuole and slender finger-like projections (acanthopodia) arising from the cytoplasm were present.

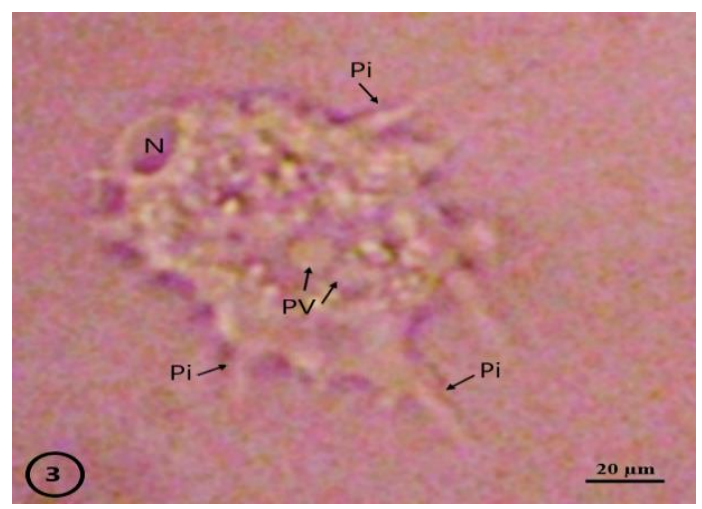

Fig. (3): Photomicrograph of Acanthamoeba sp. B trophozoite, stained with Lugol's iodine. It had an irregular outline. A single vesiculate nucleus (N) had a large centrally located dense nucleolus, surrounded by a clear halo and a thin nuclear membrane. The trophozoite was also characterized by having slender finger-like cytoplasmic projections (acanthopodia) (Pi) and more than contractile vacuole $(\mathrm{CV})$.

Acanthamoeba sp. B cyst

Cyst of Acanthamoeba sp. B (Fig. 4) had a characteristically stellate shape. It was 14-16 $\mu \mathrm{m}$ in size, lie within a wall. The endocyst was stellate and often had six pores, while ectocyst was wavy. Five rays were arranged into two isosceles.

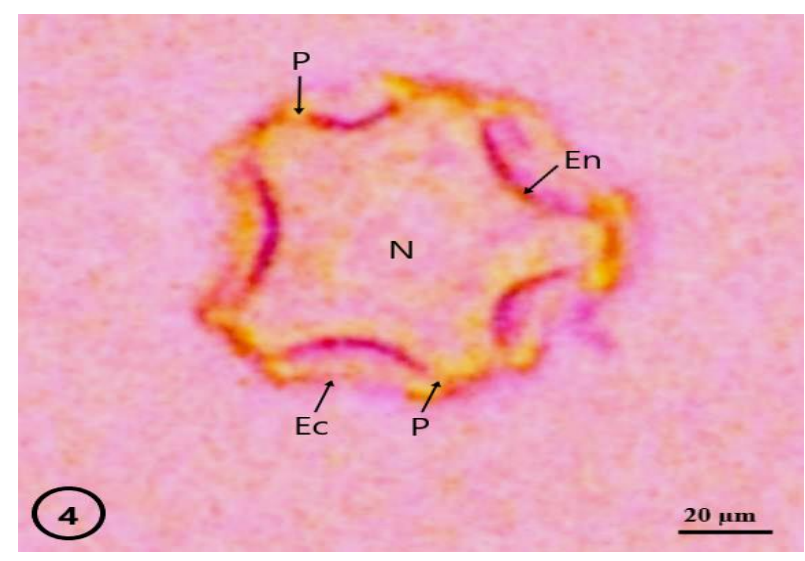

Fig. (4): Photomicrograph of Acanthamoeba sp. B cyst, stained with Lugol's iodine. It had a single nucleus (N). Endocyst (En) usually with pronounced polygonal and with mainly five rays, which appearing possibly wider at the tip. Ectocyst (Ec) was transparent and did not apply on the endocyst. 


\section{Molecular study}

Only two specimens from each of the two morphologically identified Acanthamoeba sp. A and Acanthamoeba sp. B were subjected to the molecular study by a simple PCR technique, using the genus-specific primer for Acanthamoeba to confirm the identification and to identify the species name. Results showed that the morphologically identified Acanthamoeba spp. gave $100 \%$ positivity, and when applied to agarose gel electrophoresis, the two Acanthamoeba spp. appeared at $450 \mathrm{bp}$ (Fig. 5). One major genotype was identified, with two subclusters T4A and T4B and were submitted to the gene bank under accession no. MT944118 and MT944119.

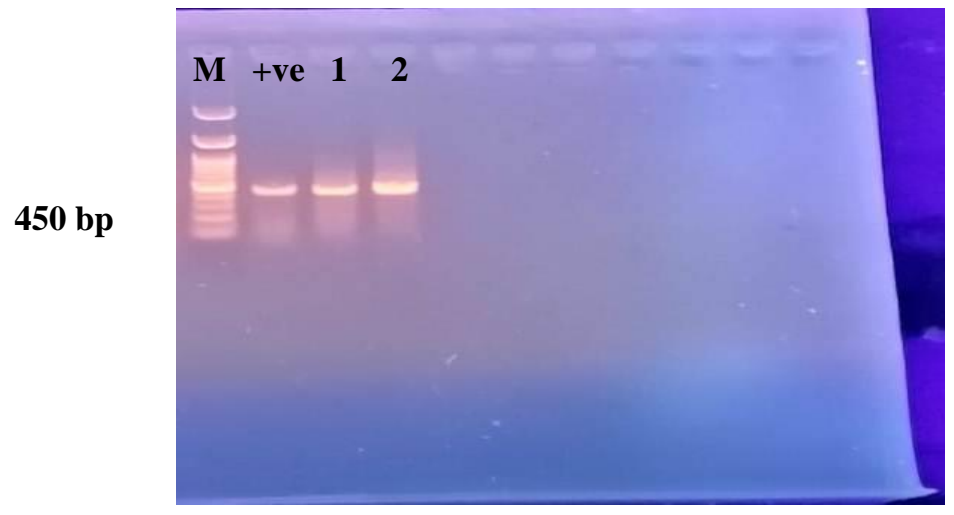

Fig. (5): Agarose gel electrophoresis of PCR amplified product of DNA of Acanthamoeba spp. Lane 1: marker; Lane 2: control positive; Lanes 1 and 2: positive samples.

\section{DISCUSSION}

In the present study, 59 (53.63) out of 110 tap water samples collected from Nasr City, Cairo, Egypt were found containing members of pathogenic FLAs belonging only to genus Acanthamoeba. Several studies on isolated Acanthamoeba spp. from tap water in Egypt have been carried out: Hikal et al. (2015) isolated Acanthamoeba spp. from 36 (72\%) out of 50 tap water samples from Dokki, Giza. Al-Herrawy et al. (2013) isolated Acanthamoeba spp. from 211 (58.6\%) out of 360 tap water samples from different localities in Delta region. Tawfeek $\boldsymbol{e t}$ al. (2016) detected Acanthamoeba spp. from 11 (31.4\%) out of 34 tap water samples in Giza. In other countries as United Kingdom, Kilvington et al. (2004) recorded an incidence of $26.9 \%$ Acanthamoeba spp. from tap water samples collected from Bibby Sterilin, Staffordshire. In Uganda, Sente et al. (2016) found Acanthamoeba spp. from 36 (42.9\%) out of 88 tap water samples. In Iran, Acanthamoeba spp. have been isolated from 45 (48\%) out of 94 tap water samples (Bagheri et al., 2010). In Spain, Lorenzo-Morales et al. (2005) detected Acanthamoeba spp. in $88(59.5 \%)$ out of 148 tap water samples. In Italy, Di Filippo et al. (2015) found Acanthamoeba spp. in 15 (17.8\%) out of 48 tap water samples. In Brazil, Carlesso et al. (2010) isolated Acanthamoeba spp. from $73.8 \%$ of the examined drinking water. In Ohio, USA, Stockman et al. (2011) morphologically identified Acanthamoeba spp. in $237(51 \%)$ of households' samples. Difference in the prevalence of Acanthamoeba spp. in tap water samples in 
different countries might be due to the difference in the tap water hygiene in each country (Jeong and Yu, 2005). Whereas, Hassan et al. (2012) stated that, the difference in detection rates of Acanthamoeba spp. in different countries and localities might be influenced by raw water sources either fed by roof-top tanks or directly from water pipes.

The present Acanthamoeba spp. were morphologically identified on bases of the characteristics of trophozoites and cysts according to Pussard and Pons (1977) and Page (1988). The current trophozoites were characterized by slender finger-like projections (acanthopodia) arising from cytoplasm and possessed a distinctive large nucleolus and more than contractile vacuole. While the present cysts were characterized by their clearly distinguished double cyst walls; an outer ectocyst and an inner endocyst. Several authors used the same criteria of trophozoites and cysts for the differentiation between Acanthamoeba spp. and the other pathogenic free-living amoebae (e.g., Al-Herrawy, 1992; Schuster and Viseversa, 2004; Salahuldeen et al., 2020). Mature cyst had been previously described (Mattar and Byers, 1971), as it was spherical, with a double wall; an outer exocyst that was continuous over the entire surface of the cyst, and an inner endocyst that was discontinuous in areas referred to as the ostioles.

In the current study, only two Acanthamoeba spp. were morphologically recognized as Acanthamoeba A and Acanthamoeba B from tap water samples in Nasr City, Cairo, Egypt. Acanthamoeba spp. look remarkably similar, when microscopically viewed, but careful observation (especially on the cyst stage) led to several species being described, and a classification system was created (Pussard and Pons, 1977). This scheme has fallen out of favor with some workers because of the realization that cyst morphology depends on the conditions in which they were created (Stratford and Griffiths, 1978). Other workers suspected that the twenty or so species named based on morphology were unlikely to be valid and attempts were made to use isoenzyme analysis to classify genus Acanthamoeba (Costas and Griffiths, 1984).

The two morphologically identified Acanthamoeba spp. studied herein proved to be members of the same genus, when were molecularly tested using a simple PCR technique. One major genotype was identified (T4), with two subclusters T4A and T4B and was submitted to the gene bank under accession No. MT944118 and MT944119. In Egypt: Al-Herrawy et al. (2015) found only $12(25 \%)$ of the morphologically identified Acanthamoeba spp. proved to be species of genus Acanthamoeba after molecular conformation using PCR technique. Also, in Egypt, Morsy et al. (2016) molecularly investigated Acanthamoeba spp. using PCR in $82.6 \%$ of the examined tap water samples, proved to be morphologically positive for the same genus.

\section{REFERENCES}

Abdul Majid, M.A.; Mahboob, T.; Mong, B.G. J.; Jaturas, N.m.; Richard, R.L.; Tian-Chye, T.; Phimphila, A.; Mahaphonh, P.; Aye, K.N.; Aung, W.L.; Chuah, J.; Ziegler, A.D.; Yasiri, A.; Sawangjaroen, N.; Lim, Y. A. L. and Nissapatorn, V. (2017). Pathogenic waterborne free-living amoebae: An update from selected Southeast Asian countries. Plos One, 12: ID e0169448.

Al-Herrawy, A.Z. (1992). In vitro cultivation of agents of amoebic meningoencephaiitis isolated from water and sewage. Ph.D. Thesis, Fac. Vet. Med., Alexandria University.

Al-Herrawy, A.Z.; Bahgat, M.; Mohammed, A.; Ashour, A. and Hikal, W. (2013). Morphophysiological and biochemical criteria of Acanthamoeba spp. isolated from the Egyptian aquatic environment. Iran. J. Parasitol., 8: 302-312. 
Al-Herrawy, A.Z.; Heshmat, M.G.; Abu Kabsha, S.H.; Gad, M.A. and Lotfy, W.M. (2015). Occurrence of Acanthamoeba species in the Damanhour drinking water treatment plant, Behera Governorate (Egypt). Rep. Parasitol., 4: 15-21.

Anwar, A.; Numan, A.; Siddiqui, R.; Khalid, M. and Khan, N.A. (2019). Cobalt nanoparticles as novel nanotherapeutics against Acanthamoeba castellanii. Parasites \& Vectors, 12:1-10.

APHA (2005). Standard methods for the examination of water and wastewater 21st ED, APHA, WEF and AWWA, Washington, DC.

Badirzadeh, A.; Niyyati, M.; Babaei, Z.; Amini, H.; Badirzadeh, H. and Rezaeian, M (2011). Isolation of free-living amoebae from Sarein hot springs in Ardebil Province, Iran. Iran. J. Parasitol., 6: 1-8.

Bagheri, H.; Shafiei, R.; Shafiei, F. and Sajjadi, S. (2010). Isolation of Acanthamoeba spp. from drinking waters in several hospitals of Iran. Iran. J. Parasitol., 5: 19-35.

Carlesso, A.; Artuso, G.; Caiimo, K. and Rott, M. (2010). Potentially pathogenic Acanthnmoeba isolated from a hospital in Brazil. Curr. Microbiol., 60: 185-190.

Costas, M. and Griffiths, A.J. (1984). The esterases and acid phosphatases of Acanthamoeba (Amoebida, Acanthamoebidae). Protistol., 20: 33-41.

Culbertson, C.G.; Smith, J.W. and Minner. H. (1958). Acanthamoeba: observations on animal pathogenicity. Science, 27 (3313):1506.

Culbertson, C.G.; Smith, J.W.; Cohen, I. and Minner. J.R. (1959). Experimental infection of mice and monkeys by Acanthamoeba. Am. J. Pathol., 35: 185-197.

Di Filippo, M.M.; Santoro, V.; Lovregli, P.; Monno, R.; Capolongo, C.; Calia, C.; Fumarola, L.; D'Alfonso, R.; Berrilli, F. and Di Cave, D. (2015). Isolation and molecular characterization of free-living amoebae from different water sources in Italy. Int. J. Environ. Res. Public Health., 12: 3417-3427.

Greub G. and Raoult D. (2004). Microorganisms resistant to free-living amoebae. Clin. Microbiol. Rev., 17: 413-433.

Hassan, A.; Farouk, H.; Hassanein, F.; Abdul-Ghani, R.; Abdelhady, A.H. (2012). Acanthamoeba contamination of hemodialysis and dental units in Alexandria, Egypt: A neglected potential source of infection. J. Infect. Public Health, 5: 304-310.

Helling, R.B.; Goodman, H.M. and Boyer, H.W. (1974). Analysis of R. EcoRI fragments of DNA from lambdoid bacteriophages and other viruses by agarose- gel electrophoresis. J. Virol., 14: 1235-1244.

Hikal, W.; Zaki, B. and Sabry, H. (2015). Evaluation of ozone application in dental unit water lines contaminated with pathogenic Acanthamoeba. Iran. J. Parasitol., 10: 410-419.

Jeong, H. and Yu, H.: (2005). The role of domestic water in Acanthamoeba contamination in contact lens storage cases in Korea. Korean. J. Parasitol., 43: 47-50. 
Kilvington, S.; Gray, T.; Dart, J.; Morlet, N.; Beeching, J.; Frazer, D. and Matheson, M. (2004). Acanthamoeba keratitis: the role of domestic tap water contamination in the United Kingdom. Invest. Ophthalmol. Vis. Sci., 45: 165-169.

Kinde, H.; Read, D.H.; Daft, B.M.; Manzer, M.; Nordhausen, R.W.; Kelly, D.J.; Fuerst, P.A.; Booton, G. and Visvesvara, G.S. (2007). Infections caused by pathogenic free-living amebas (Balamuthia mandrillaris and Acanthamoeba sp.) in horses. J. Vet. Diagn. Invest., 19: 317-322.

Kumar, S.; Stecher, G. and Tamura, K. (2016). MEGA7: Molecular evolutionary genetics analysis version 7.0 for Bigger Datasets. Mol. Biol. Evol., 33: 1870-1874

Lorenzo-Morales, J.; Lindo, J.F.; Martinez, E.; Calder, D.; Figueruelo, E. and Valladares, B. (2005). Pathogenic Acanthamoeba strains from water sources in Jamaica, West Indies. Ann. Trop. Med. Parasitol., 99: 751-758.

Martinez, A.J. and Visvesvara, G.S. (1997). Free-living, amphizoic and opportunistic amoebae. Brain Pathol., 7: 583-598.

Mattar, F.E. and Byers, T.J. (1971). Morphological changes and the requirements for macromolecule synthesis during excystment of Acanthamoeba castelianii. J. Cell Biol., 49: $507-519$.

Morsy, G.H.; Al-Herrawy, A.Z.; Elsenousy, W.M. and Marouf, M.A. (2016). Prevalence of free-living amoebae in tap water and biofilm, Egypt. Res. J. Pharmaceut., Biol. Chem. Sci. RJPBCS., 7: 752-759.

Neelam, S., and Niederkorn, J.Y. (2017). Pathobiology and immunobiology of Acanthamoeba Keratitis: Insights from animal models. Yale J. Biol. Med., 90: 261-268.

O'Day D.M. and Head, W.S. (2000). Advances in the management of keratomycosis and Acanthamoeba keratitis. Cornea, 19: 681-687.

Page, F.C. (1988): A new key to freshwater and soil Gymnamoebae. Freshwater Biol. Ass., Ambleside, 3-170.

Pussard, M. and Pons, R. (1977). Morphologie de la paroi Kystique et taxonomie du genre Acanthamoeba (Protozoa, Amoebida). Protistologica, 13: 557-598.

Rodriguez-Zaragoza, S. (1994). Ecology of free-living amoebae. Crit. Rev. Microbiol., 20: 225-241.

Salahuldeen, A.; Abd El-Aziz, A.; Gad, M.A.; Abou El-Nour, M.F.; Al-Herrawy, A.Z. (2020). Potentially pathogenic free-living amoebae in fresh and sea waters from Alexandria, Egypt. Egypt. J. Aquatic Biol. Fish., Vol. 24: 307-318.

Scheid, P. (2007). Mechanism of intrusion of a microspordian-like organism into the nucleus of host amoebae (Vannella sp.) isolated from a keratitis patient. Parasitol. Res., 101: 1097-1102.

Schroeder, J.M.; Booton, G.C.; Hay, G.; Niszl, I.A.; Seal, D.V.; Markus, M.B.; Fuerst, P.A. and Byers, T.J. (2001). Use of subgenic $18 \mathrm{~S}$ ribosomal DNA and sequencing for genus and genotyping identification of Acanthamoeba from humans with keratitis and from sewage sludge. J. Clin. Microbiol., 39: 1903-1911. 
Schuster, F.L. and Visvesvara, G.S. (2004). Free-living amoebae as opportunistic and nonopportunistic pathogens of humans and animals. Int. J. Parasitol., 34: 1001-1027.

Sente, C.; Erume, J.; Naigaga, I.; Magambo, P.K.; Ochwo, S.; Mulindwa, J. ; Namara, B.G.; Kato, C.D.; Sebyatika, G.; Muwonge, K. and Ocaido, M. (2016). Occurrence and genetic characterisation of Acanthamoeba spp. from environmental and domestic water sources in Queen Elizabeth Protected Area, Uganda. Parasites \& Vectors., 9:1-8.

Shyrobokov, V.; Poniatovskyi, V.; Chobotar, A. and Salamatin, R. (2020). Morphological, physiological, and genetic characteristics of protozoa of genus Acanthamoeba, isolated from different deposit of bentonite in Ukraine. Ann Parasitol., 66: 69-75.

Stockman, L.J.; Wright, C.J.; Visvesvara, G.S.; Fields, B.S. and Beach, M.J. (2011). Prevalence of Acanthamoeba spp. and other free-living amoebae in household water, Ohio, USA-1990-1992. Parasitol. Res., 108: 621-627.

Stratford, M.P. and Griffith, A.J. (1978). Variations in the properties and morphology of cysts of Acanthamoeba castellanii. J. Gen. Microbiol., 108: 33-37.

Tawfeek, G.M.; Bishara, S.A.; Sarhan, R.M.; Taher, E.E. and Khayyal, A.E. (2016). Genotypic, physiological, and biochemical characterization of potentially pathogenic Acanthamoeba isolated from the environment in Cairo, Egypt. Parasitol. Res., 115: 18711881.

Trabelsi, H.; Dendana, F.; Sellami, A.; Sellami, H.; Cheikhrouhou, F.; Neji, S.; Makni, F. and Ayadi, A. (2012). Pathogenic free-living amoebae: epidemiology and clinical review. Pathol. Biol. (Paris), 60: 399-405. 